\title{
Multi Agent Systems for Multi Enterprise Scheduling
}

\author{
Dieter Spath, Gisela Lanza, Markus Herm
}

Institute for Machine Tools and Production Science, Technical University Karlsruhe, Germany gisela.lanza@mach.uni-karlsruhe.de

Abstract: 'Multi Enterprise Scheduling' considers the temporal allocation of activities to resources. As soon as the specific targets and additional conditions have been achieved, the most suitable enterprise to execute the activity must be chosen. In the following article, the activity model presents the connections and logical sequence of the activities within the company overlapping business processes. The Organization units are identified in an Organizational and Resource Model. In the concept they are represented through Multi Agents, namely the Activity Agent and the Resource Agent. To meet the requirements of communication and cooperation across companies, it is attempted to implement this agent technology with web parts.

Key words: Manufacturing control and scheduling, Multi Agent System, Multi-Enterprise Scheduling, Business Process Modelling

\section{INTRODUCTION}

The demand for distributed value added nets [1] requires the conversion of dynamic process chains across several autonomous companies. Nowadays these so called Supply Chains are no 'chains' anymore, they occur as supply networks (see Figure 1).

In these dynamic supply networks, new optimization potentials must be developed through considering the involvement of a company with its customers and suppliers [2].

With virtual value added networks, or rather 'virtual companies', these partnerships are subjected to a relatively fast change. Thus, it is necessary 
for optimization to occur throughout the entire value added chain, that is to surpass the company's boundaries.
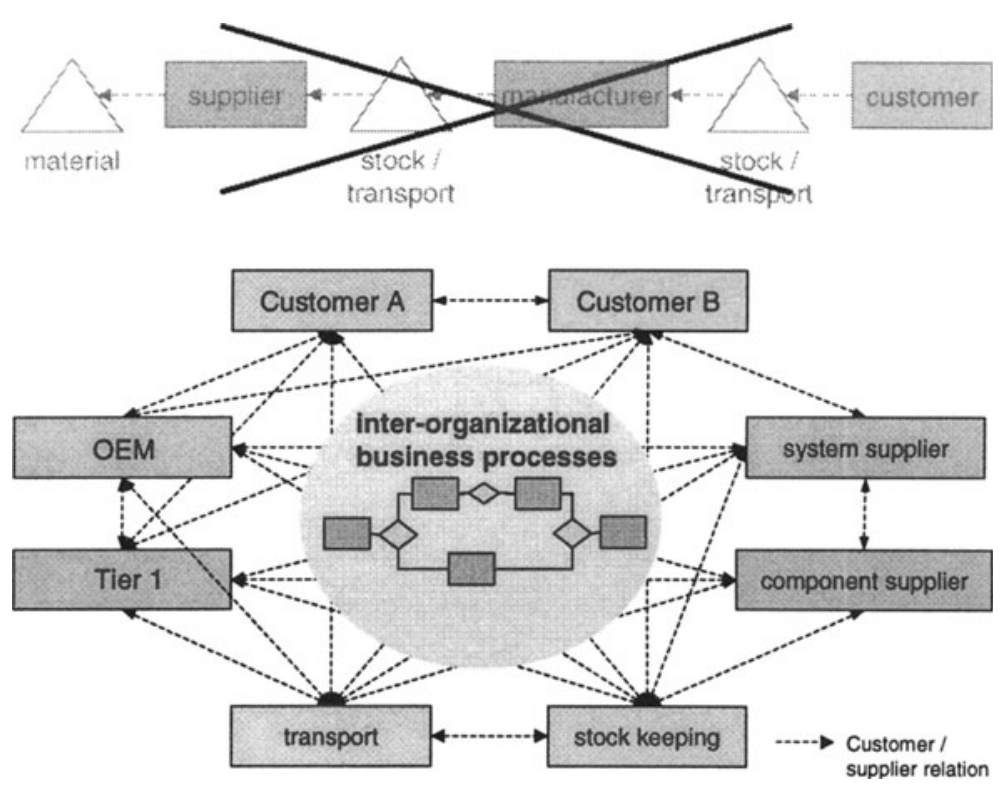

Figure 1: From Supply Chain towards Supply Network

\subsection{Motivation}

If one considers traditional solutions, the planning of the supply chain is initially strongly oriented towards production planning. Taking a rough plan as a starting point, which is then systematically dismantled into more refined plans up to the final production planning.

For this, Enterprise Resource Planning (ERP) Systems for materials management and production planning are introduced into companies. They are, however, not designed to optimize company overlapping supply chains in a decentralized way. They take fixed capacity quantities and production times as a basis.

It can be observed that stock transparency does not exist throughout the entire supply network, since standardized mapping of operative business processes is not available [3]. 


\subsection{Scheduling Approach}

The focal point of this article is that production processes are eventorientated and subject to certain interferences. This event-orientation is intensified when stock keeping and transport have to be regarded as the determining factors of schedule reliability (see Figure 2).

Consequently, only decentralized planning approaches can achieve a certain schedule stability, because local solvable deviations must no longer be taken into account in a global planning process.

This requires an event-oriented and decentralized scheduling approach of the company overlapping business processes. The presented solution with Multi Agent Systems meets these requirements. An uncomplicated adaptability at structural changes is achieved through the autonomous negotiation of the multi agents. Moreover a parallel scheduling is possible on distributed systems. To meet the requirements of communication and cooperation across companies, it is attempted to implement agent technology via web parts.

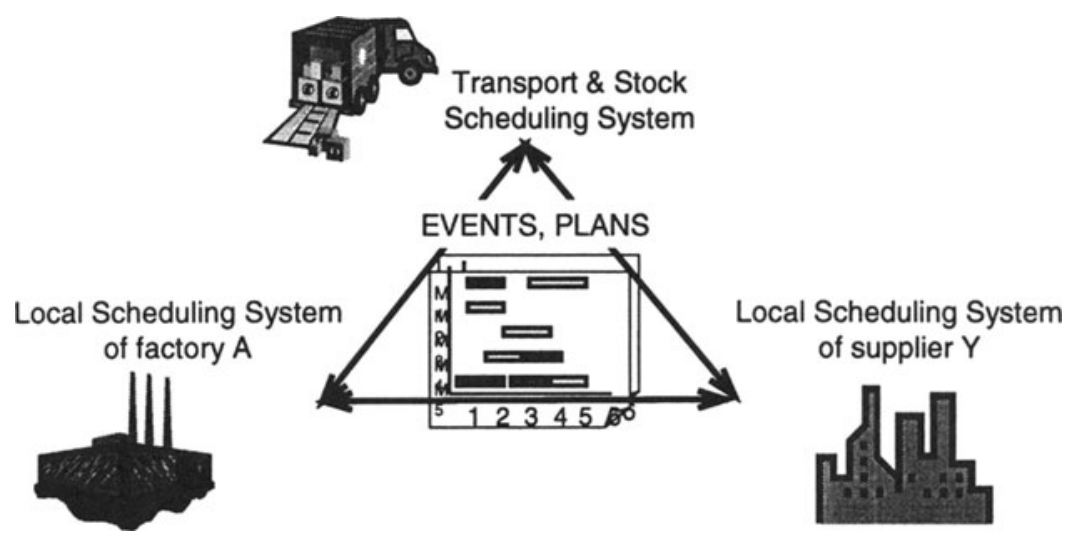

Figure 2: Multi-Enterprise Scheduling Scenario

\section{MODELING OF BUSINESS OVERLAPPING PROCESSES - ACTIVITY MODEL}

The objective of the project is to generate an optimized and realizable operation plan, in which the diverse influential factors and restrictions are taken into consideration. An abstract model of the interorganizational processes is required to develop solutions for the scheduling problem. The basic activity model [4] presents the connections and the logical sequence of 
the activities in the regarded process. The modeling of activities is done through the generation of activity components (Enterprise Activities), which contains a status model as well as through the construction of rules (Business Rules), which are situated between these components [5].

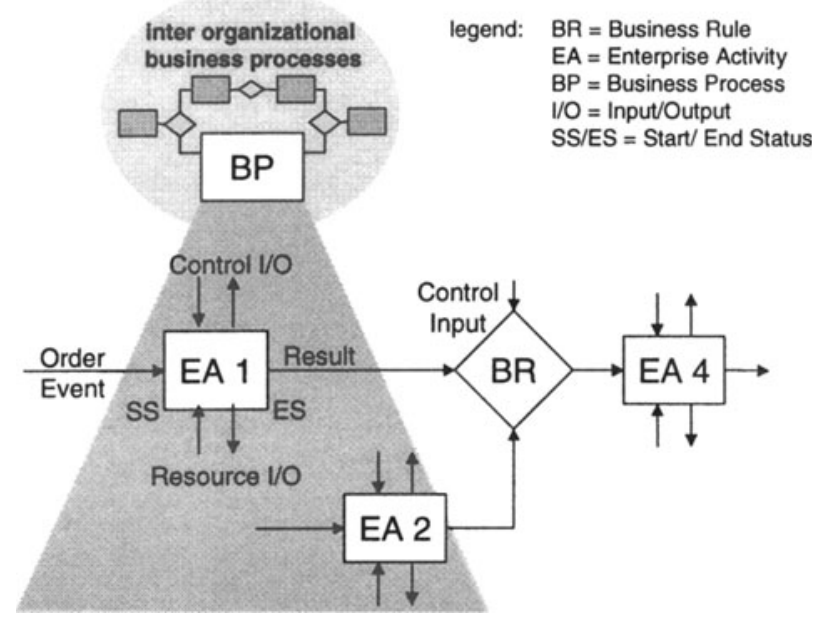

Figure 3: The basic Activity Model of the Multi Enterprise Scheduling

For the Multi Enterprise Scheduling the activity model is characterized by the following objects and relations:

Orders for the product manufacturing: For products, various manufacturing alternatives with different production steps (work schedules) and alternative usable resources can exist. Orders are mapped in the model through Event-Input, which activates an Enterprise Activity. Here orders are defined through order number, start status, variant, batch number, input date, output date, prioritization and target costs.

Resources such as machines, raw materials, personnel, as well as warehouse and transport type, which are generally available for several products. The current capacity situation and future capacity changes must be considered in the optimization. Mapped in the model through Resource Input (Information from the resource pool, whether the required resources are available) and Resource Output (Resource de-allocation after termination or interruption); described through resource number, resource type (internal, external resource), resource class, availability, allocation and cost rate per unit of time.

Interferences of a technical or an organizational type - for example the loss of resources leading to modifications in the planning environment, which must be responded to appropriately. Mapped through: Control Input (interference signal or signal at resource reclaim) and Control Output 
(activation of alternative processes, for example reworking with interferences).

Constraints, that have to be (un)conditionally maintained and which are mostly technical, such as manufacturing specifications or the restriction of a double reservation of resources or a warehouse safety stock, a minimum batch size, etc. Mapped in the model through Business Rules, which describe the conditions under which the various activities are started.

Objectives such as strict schedule compliance, cost optimization, maximum plant utilization with maximum flexibility (robustness versus order variations), constant capacity utilization, minimum processing time, etc.

Optimization of scheduling is controlled through these constraints and objectives. The restrictions define the solution space. Via the objectives, the quality of the solution can be generated .

\section{ORGANIZATIONAL AND RESOURCE MODEL}

Why an organizational model? The structuring of business processes, as well as the allocation of the resources, can be clearly presented through the internal and intermediate organizational structures across collaborating companies.

In abstract terms, the core elements of company organizations are organizational units as well as relationships between them.

\subsection{Organizational Model}

The organizational model described here [6], considers the internal hierarchical structure as well as the intermediate net-like dimension. The latter gains increasingly in importance through the above-mentioned demands.

Hierarchical structures are the usual form of internal organizational structure, since they are ideal for the unambiguous definition of: power of decisions, instructions and control as well as supervisory duties. Hierarchical structures form the basis of this model. For this purpose, the organizational units are defined according to resource-oriented aspects and sorted into a hierarchy. Possible organizational units are for example, the entire company, suppliers, transport companies, sites, manufacturing areas, warehouse areas or machine groups.

Complex organizational forms are not, however, exclusively organized hierarchically. This applies in particular to legitimate and economically independent companies. The relationships between these include neither the 
'power of decision' nor the 'authority to instruct', but they place the aspect of coordination and communication in the foreground. Thus the networklike, tendentiously dynamic dimension of the coordinating, logistic relationships are also considered on all levels in the hierarchy, as well as the hierarchical, or rather static dimension of the internal main administration system in the organizational model.

In the organizational model (see Figure 4), this overlay of the hierarchical and net-like structures is represented through two different types of relationship. Both types are orientated (Arrow) and define a potential use of an organizational unit by another one. The first can be described in the following as a customer and the other as a supplier. For example, a company (Arrow-source) loads an assignment (Relationship-arrow) in a specific plant (Arrow-destination).

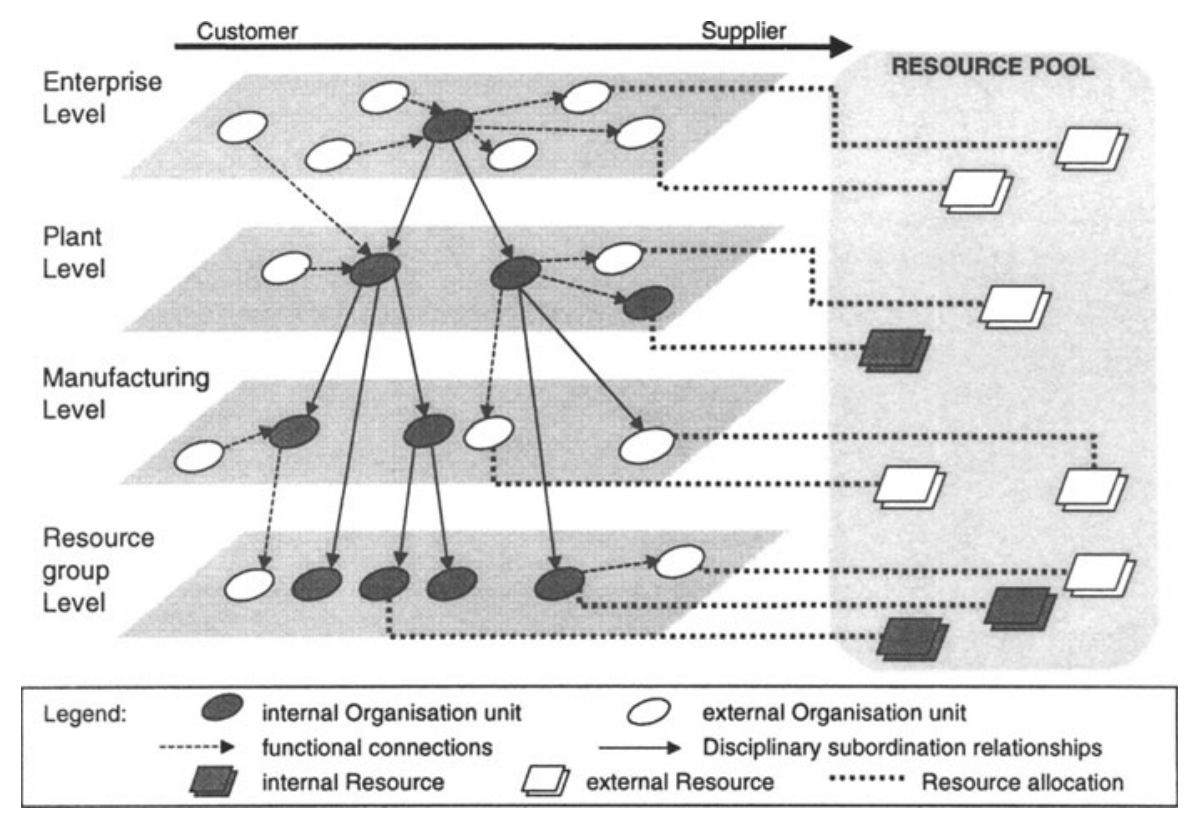

Figure 4: Organization and Resource Model with organization units and allocated resources.

\subsection{Resource Allocation - Generation of a Resource Pool}

The available resources are now allocated to the organizational units [7]. Resources for example, are employees, production systems, test beds, ...

In figure 1 the resources are coupled through connecting lines to the respective organizational units. 
Coupling of the Activity Model with the Organization and Resource Model occurs because each individual activity out of the activity model is only processed through one organizational unit. If this organizational unit is an external one, for example a supplier, then the required resources will be administered by this organizational unit and must be inquired about there. The more detailed these activities are modeled, the more exactly the allocation of the executive organizational units and resources can occur.

The static capacity of a superordinate organizational unit is the sum of the respective resources of the disciplinary subordinate units. The sum is referred to as internal resources.

Resources of other organizational units can be used in the same way, for example the use of a test bed or the formation of a worker team. These combinations are marked through the functional connections in the organizational model. The subsequent useable resources are allocated us external resources of the superordinate organizational unit.

The Organizational and Resource Model administers the entire supply of the resources, which are available in a resource pool. Within this pool, one distinguishes between the company internal resources (sum of all internal resources) and company external resources, that represent the partial quantity of the external resources, which do not refer to other organizational units in the same company, but to external sources. The external resource allocation needs a specific communication and coordination, since arranged parameters (Priorities of the assignment, costs, etc.) have to be negotiated, in order to avoid, for example, double reservations

\section{MULTI AGENTS FOR SCHEDULING}

For the scheduling of activities which need these external resources the requirements like 'information exchange', 'Negotiation' and 'Task' must be satisfied. These demands shall be achieved via three characteristics of the multi agent technology: The agent architecture, the agent interface (especially the agent language) and the agent platform ( see Figure 5)

\subsection{Agent Architecture}

The agent architecture enables the autonomous, or rather partly autonomous, implementation of decisions and tasks. The agent makes assumptions about its environment (belief), it possess an objective function (desire) and an intention module (intention), in which it makes plans of action or decisions as to how it wants to adapt its environment to the target status (corresponds to BDI-Architecture [8]). 
Based on the coupling of the two models every identified Organization unit is represented by two agent types with specific assignments.

The Activity Agent (or Order Agent) attempts to make activities that require external resources, capable of starting. Visualized through the functional connection in the Organization Model. Therefore the activity agent negotiates with the suppliers' agent (from the resource agent type) about the resource offers, under specific target restrictions. The most important assignments are dispatching enquiries, evaluating offers and eventually assigning orders.

The Resource Agent of an organization unit is interested in a constant use of its resource and pursues this objective, this means that it provides free capacities of its resource to the agents of the customers (from the activity agent type) and negotiates with them. It must take current allocation dates as a basis.

Additionally there are the agents of the customers and suppliers, which are registered (known) on the agent platform.

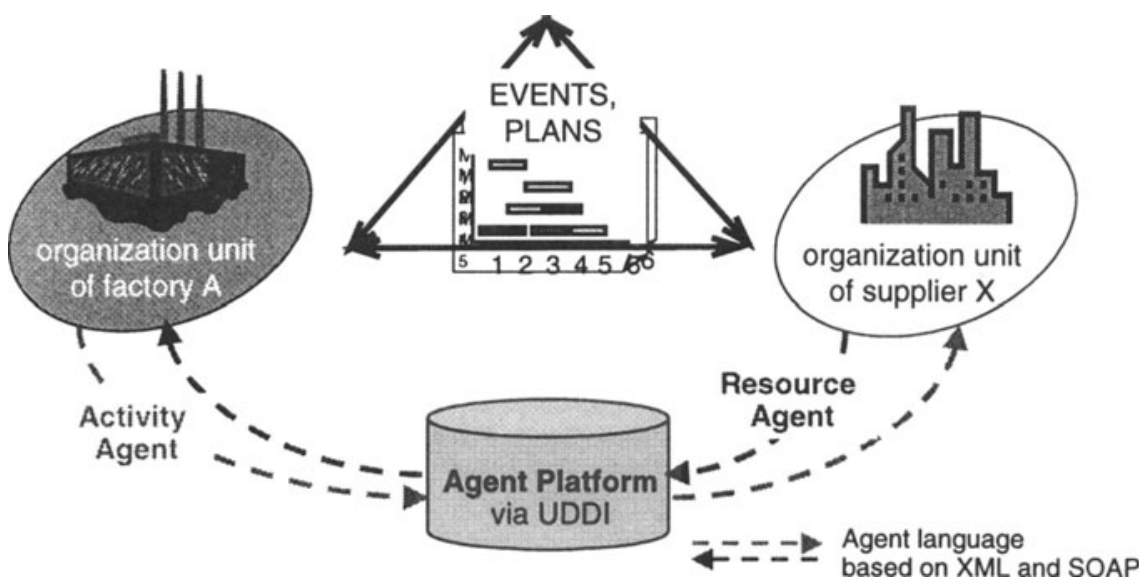

Figure 5: Multi Agents for Scheduling

\subsection{Agent Interface}

The agent interface permits the exchange of the agents' messages between systems on the same level, via an application independent agent language. For this, the agent uses so-called 'Speech act' with which it can express its objectives to other agents. For supply chain management, five categories of speech act are defined [9] (see Table 1)

The content of the message has an application independent semantic, due to it being defined as XML schemata. The agent language is developed on 
the basis of the internet standards XML and SOAP [10]. Through the Remote Procedure Calls (RPC), the Simple Open Access Protocol (SOAP) that is also based on XML, enables applications or modules to be contacted and executed via the internet. When using both protocols together they enable distributed and platform-independent communication and releasing of tasks.

\begin{tabular}{|l|l|}
\hline Classes of speech acts & Meaning \\
\hline Information transfer & $\begin{array}{l}\text { The company-external sender delivers information to the } \\
\text { recipient. He does not combine the execution of a task. } \\
- \text { Resource availability: the enquired resource is reported as } \\
\text { being available and then booked by the resource manager. } \\
- \text { Interferences are directly reported to the event manager. }\end{array}$ \\
\hline Information inquiry & $\begin{array}{l}\text { The receiver requests specific information about a specific state } \\
\text { of affairs. } \\
\text { The resource input of an activity is enquired about. }\end{array}$ \\
\hline Negotiation & $\begin{array}{l}\text { Sender and recipient negotiate about variables (for example } \\
\text { price, delivery date, quantity to be delivered) }\end{array}$ \\
\hline Task & $\begin{array}{l}\text { The sender combines with the message the execution of a task } \\
\text { at the recipient (for example contracting) }\end{array}$ \\
\hline Error handling & routines, if no speech act is accomplished \\
\hline
\end{tabular}

Table 1: Classification of speech acts for the Supply Chain Management

\subsection{Agent Platform}

The agent platform presents the environment, on which agents can be executed. The approach is currently followed to realize the agent platform according to the experience with distributed database application and to the UDDI efforts based on XML and SOAP.

The Universal Description, Discovery and Integration (UDDI) standard creates a platform-independent, open framework for describing services, discovering businesses, and integrating business services using the Internet [11].

The principle of telephone directories vividly illustrates the procedure: the section of the UDDI-index corresponding to the normal "white" telephone directory contains the names of the companies, the addresses and standard contact information (business entity element). The "yellow pages" provide information about the products and services, which the company offers (business service element). Finally, the new "green pages" inform about the protocols that the companies use in the electronic business commerce (binding template element).

In our scenario the call of a service or order, which is registered in the database, operates approximately as follows (see Figure 6): 
An UDDI client, an activity agent of the customer Y traces the business entity information of the desired service via an inquiry in the business repository. The agent requests detailed information regarding the business service element. It negotiates with the UDDI client of the addressed company, for example the resource agent of supplier $\mathrm{X}$, through standardized speech act according to its objectives, for example target costs and delivery date. If the objectives are achieved, the assignment is processed with the help of the binding template element, for example Purchase Order Confirmation (POC) and delivery schedule. Furthermore, a special authentication mechanism guarantees that only authorized clients may apply the web services.

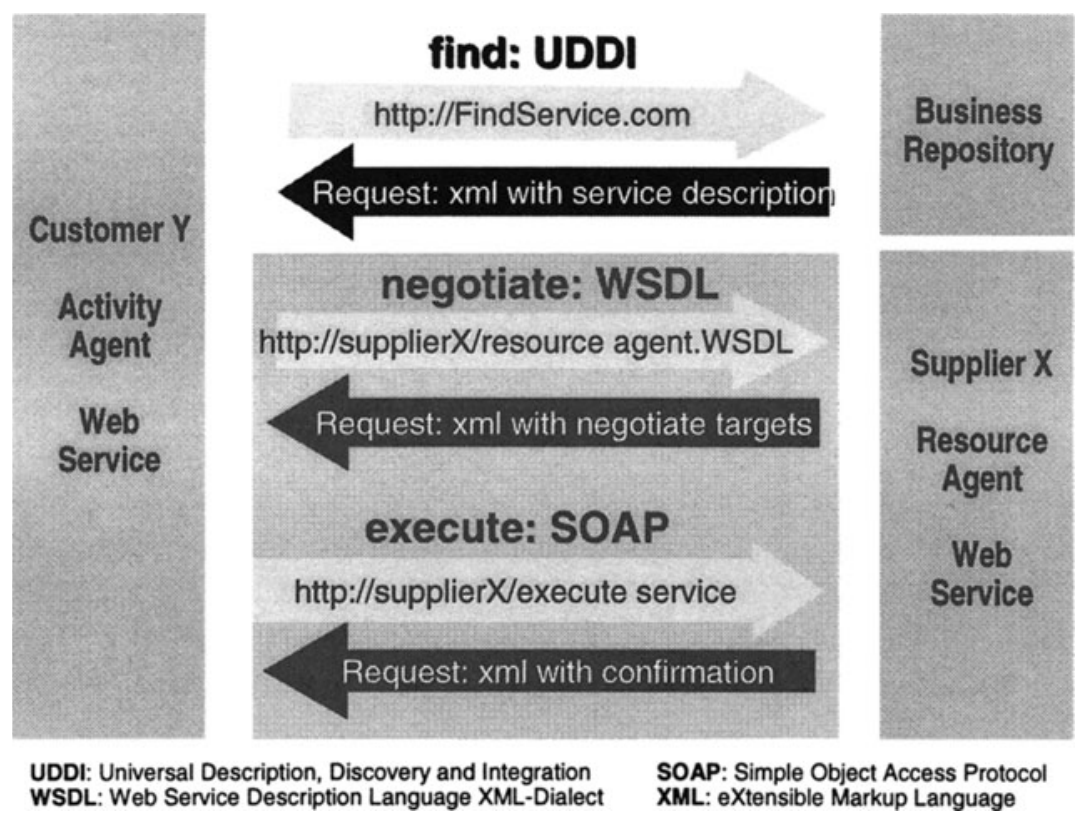

Figure 6: Agent negotiation via web services

\section{SUMMARY}

The report presents an activity model that is connected with the responsible organizational units. It presents the company overlapping business processes, which considers, in addition to production processes, stock keeping and transport as determining factors of schedule reliability.

Distributed production and logistic networks generate a higher level of coordination requirement. The presented Organizational and Resource 
Model maps these problems through the introduction of explicit communication relationships.

In the joint project 'Distributed modeling, simulation and control of business processes with generic Petri nets' supported by the ministry for economic affairs of 'Baden Württemberg', very positive results were experienced during the modeling and simulation of the Activity Model as well as the Organizational and Resource Model.

With the help of multi agents, the multi enterprise scheduling that is presented in this article, is now in the development stage: To produce a prototype there must be a specification of the negotiation protocols. Current research focuses on the development of the UDDI standards and investigates whether the above-performed scenario is realizable.

\section{REFERENCES}

[1] Scheer, A.-W.; Borowsky, R., 1999, Supply Chain Management: Die Antwort auf neue Logistikanforderungen, LM'99-Intelligente I+K Technologien, Springer Verlag, Bremen.

[2] Schönsleben, P., 1998, Integrales Logistikmanagement: Planung und Steuerung von umfassenden Geschäftsprozessen, Springer, Berlin, Heidelberg.

[3] Tiemeyer, E., 2000, Supply Chain Management - Konzeptentwicklung und Softwareauswahl in der Praxis, atp 42, Heft 10: 24-32.

[4] Kühnle, H., Sternemann, K.-H., Harz, K., 1998, Herausforderung Geschäftsprozesse Den Wandel organisatorisch und technisch gestalten, Logis Verlag GmbH, Stuttgart.

[5] CIMOSA - Open System Architecture for CIM; ESPRIT Consortium AMICE, Springer Verlag 1993, ISBN3-540-56256-7, ISBN 0-387-56256-7, Heidelberg.

[6] Appelrath, H.-J., Sauer, J., Fresse, t., Teschke, T., 2000, Strukturelle Abbildung von Produktionsnetzwerken auf Multiagentensysteme, KI Künstliche Intelligenz, Heft 3/200: 64-70, arenDTaP Verlag, Bremen.

[7] Spath, D.; Sternemann; K.-H.; Lanza, G.: Supply Network Simulation. CIRP Proceeding of the 34th International Seminar for Manufacturing Systems, Athens 2001.

[8] Rao, Anand S.; Georgeff, Michael P.; Sonenberg; 1995, BDI Agents: from Theory to Practice, Proceeding of the First International Conference on Multi-Agent Systems, pp 312-319, MIT Press, MA.

[9] Stiefbold, O., 1998, Konzeption eines reaktionsschnellen Planungssystem für Logistikketten auf Basis von Software-Agenten, Forschungsberichte aus dem Institut für Werkzeugmaschinen und Betriebstechnik der Universität Karlsruhe, Dissertation, ISSN 0724-4967, Karlsruhe.

[10] W3C- specification, 2001, SOAP Simple Object Access Protocol, http://www.w3.org/TR/SOAP/, DATE 2001/03/10.

[11] UDDI.org, 2001, Universal Description, Discovery and Integration, http://www.uddi.org, DATE 2001/03/10. 